Journal of Quantitative Spectroscopy \&

Radiative Transfer 69 (2001) 585-604
Journal of Quantitative Spectroscopy \& Radiative Transfer

www.elsevier.com/locate/jqsrt

\title{
Parameterized scattering matrices for small particles in planetary atmospheres
}

\author{
C.J. Braak ${ }^{\mathrm{a}}$, J.F. de Haan ${ }^{\mathrm{a}}$, C.V.M. van der Mee ${ }^{\mathrm{b}}$, J.W. Hovenier ${ }^{\mathrm{a}, \mathrm{c}, *}$, \\ L.D. Travis ${ }^{\mathrm{d}}$ \\ ${ }^{a}$ Department of Physics and Astronomy, Free University, De Boelelaan 1081, 1081 HV Amsterdam, The Netherlands \\ bipartimento di Matematica, Università di Cagliari, Via Ospedale 72, 09124 Cagliari, Italy \\ 'Astronomical Institute "Anton Pannekoek", University of Amsterdam, Kruislaan 403, \\ 1098 SJ Amsterdam, The Netherlands \\ ${ }^{\mathrm{d}}$ NASA Goddard Institute for Space Studies, 2880 Broadway, New York, NY 10025, USA
}

Received 8 April 2000; accepted 21 May 2000

\begin{abstract}
Parameterized matrices are discussed that may be used as (single) scattering matrices for interpretations of the brightness and polarization of planetary atmospheres containing randomly oriented small particles. A number of guidelines are developed for the construction of such matrices. These guidelines are based on (i) physical conditions for the elements of a natural scattering matrix, some holding for arbitrary scattering angles and some for the exact forward and backward scattering directions only, as well as (ii) theorems for the asymptotic behavior of coefficients in expansions of the matrix elements in generalized spherical functions of the scattering angle. A set of parameterized matrices is introduced and assessed according to these guidelines. These particular parameterizations are especially useful for scattering by particles that are not large compared to the wavelength, particles in the Rayleigh-Gans domain and for a variety of irregularly shaped particles in the visible part of the spectrum. The use of parameterized matrices as scattering matrices is illustrated by deriving their elements as functions of the scattering angle from simulated measurements of the brightness and polarization of light reflected by plane-parallel atmospheres containing aggregated or spheroidal particles. In both cases, the scattering angle dependences of the original elements are retrieved in fair approximation. (C) 2001 Elsevier Science Ltd. All rights reserved.
\end{abstract}

\footnotetext{
* Corresponding author at: Department of Physics and Astronomy, Free University, De Boelelaan 1081, $1081 \mathrm{HV}$ Amsterdam, Netherlands. Tel.: + 31-20-4447956; fax: + 31-20-4447999.

E-mail address: hovenier@nat.vu.nl (J.W. Hovenier).
} 


\section{Introduction}

Comparing the results of measurements of the brightness and the state of polarization of light scattered by an atmosphere with the results of multiple-scattering calculations is very useful for obtaining information about that atmosphere, such as abundances of its constituents, vertical cloud structure, and the physical and chemical properties of cloud or aerosol particles [1-4]. Often, assumptions are made about the particle size, shape, and composition for which the $4 \times 4$ scattering matrix, $\mathbf{F}(\Theta)$, of a collection of such particles can be calculated. The scattering matrix transforms Stokes parameters of an incident beam into those of the scattered beam $[5,6]$. Such matrices then serve as input in a trial-and-error formulation including multiple scattering. In this case, measurements of brightness and polarization are interpreted in terms of physical properties of the particles such as size, refractive index, and parameters describing the exact shape (e.g., the aspect ratio in the case of spheroidal particles). Fig. 1a shows a scheme of this approach for analyzing brightness and polarization of a multi-layered atmosphere with identical or different scattering matrices in different layers. In case the particles can be assumed to be spherical, Mie theory can be applied. This approach has been very successful [2,7-10].

However, particles in nature may have many other shapes (needles, hexagonal crystals, aggregates, etc.). If no reliable a priori knowledge about the size, shape, or composition of the particles is available, or if the calculations of scattering matrices as functions of the scattering angle, $\Theta$, are prohibitively time-consuming in a trial-and-error formulation, one might use parameterized matrices as scattering matrices. This may be considered as an extension of the well-known parameterization of the phase function (the 1,1-element of the scattering matrix) as a one-term [11]
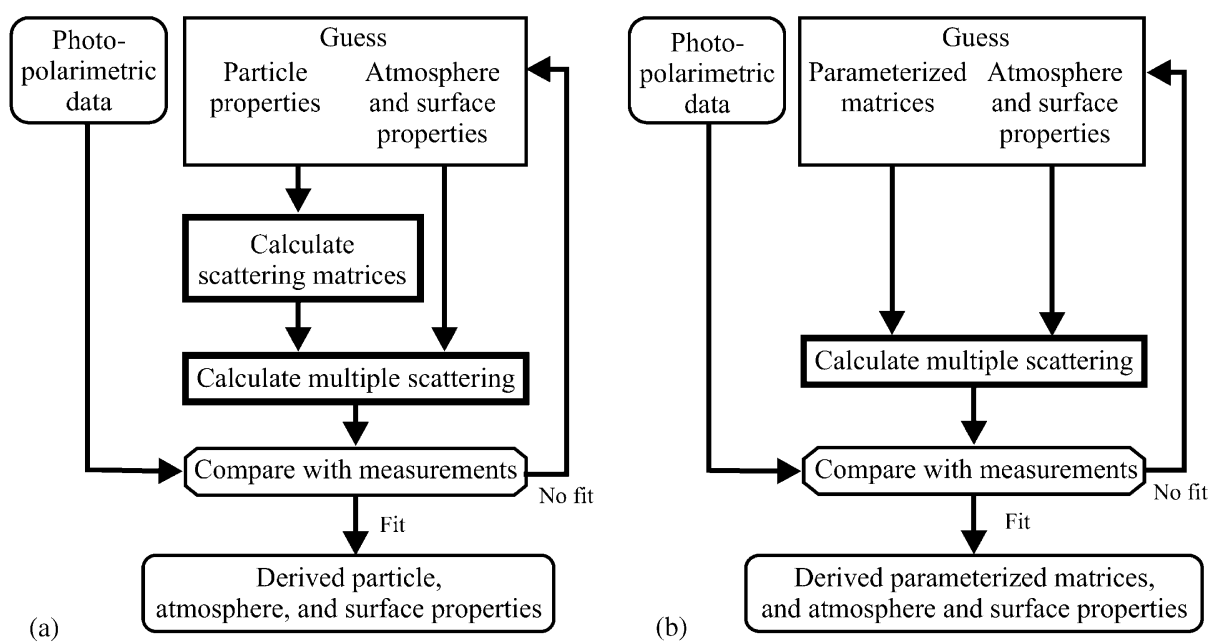

Fig. 1. Scheme showing different approaches for analyzing photopolarimetric data of a multi-layered atmosphere bounded below by a surface in a trial-and-error formalism. (a) Analysis using single-scattering algorithms. (b) Analysis using parameterized matrices instead. Particle properties are properties describing shape, size, and refractive index; atmosphere properties are properties such as optical thickness, mixing ratio, and number of layers; surface properties refer to the reflection properties of the surface. 
or two-term Henyey-Greenstein function [12]. Fig. 1b shows how photopolarimetric data of a multi-layered atmosphere can be used to determine parameter values of parameterized matrices.

Using parameterized matrices, measured quantities are interpreted in terms of the singlescattering behavior of the particles rather than their size, shape, and composition. Often, one can infer important physical constraints from derived parameterized matrices without knowing the precise shapes of the particles. For instance, the shape of $F_{11}(\Theta)$ as a function of scattering angle gives an indication of the size of the particles, and the ratios $F_{21}(\Theta) / F_{11}(\Theta)$ and $F_{22}(\Theta) / F_{11}(\Theta)$ may provide information about the sphericity of the particles. Also, one may use single-scattering algorithms or measurements to try to interpret derived parameterized matrices in terms of particle properties.

Employing terms like generalized single-scattering properties [13], synthetic phase matrix [14], and synthetic scattering matrix [15], investigators have previously adopted similar approaches for interpreting polarization measurements of the planet Saturn and its satellite Titan, without taking into account physical constraints that were later published. These approaches are briefly reviewed in Section 3. In Section 4, necessary physical constraints are described that must be imposed on parameterized matrices. We define a parameterized scattering matrix (PSM) as a parameterized matrix that meets all these constraints. In Section 5, the convergence of the expansion of parameterized matrices in generalized spherical functions is discussed. A particular parameterization that is applicable to particles that are not large compared to the wavelength, particles in the Rayleigh-Gans domain, and a variety of irregularly shaped particles in the visible part of the spectrum is discussed in Section 6. In order to illustrate the applicability of PSMs, two case studies are described in Section 7 in which PSMs are derived from simulated measurements of the brightness and polarization of sunlight scattered by atmospheres containing aggregated or spheroidal particles. We will, however, begin with a general description of scattering matrices.

\section{Structure of scattering matrices}

In its most general form, the scattering matrix of a volume element of particles at a certain wavelength and with respect to a certain reference plane consists of 16 nonzero elements that are functions of scattering and azimuthal angles [5]. However, for several important cases, its structure is much simpler. One such special case occurs when the particles are randomly oriented and occur in equal numbers as their mirror particles. Then, the scattering matrix can be written as

$$
\mathbf{F}(\Theta)=\left[\begin{array}{cccc}
a_{1}(\Theta) & b_{1}(\Theta) & 0 & 0 \\
b_{1}(\Theta) & a_{2}(\Theta) & 0 & 0 \\
0 & 0 & a_{3}(\Theta) & b_{2}(\Theta) \\
0 & 0 & -b_{2}(\Theta) & a_{4}(\Theta)
\end{array}\right],
$$

when the scattering plane acts as the reference plane. Since this special case will generally be a good approximation for an arbitrary collection of randomly oriented particles, we will use this matrix structure throughout this paper. For a collection of optically inactive homogeneous spherical particles, $a_{1}(\Theta)=a_{2}(\Theta)$ and $a_{3}(\Theta)=a_{4}(\Theta)$. 
Inequalities for arbitrary scattering angles as given in Refs. [16,17] imply that the absolute value of any of the elements of $\mathbf{F}$ cannot exceed the value of $a_{1}$, which is nonnegative. In this paper, we normalize all scattering matrices so that the average of $a_{1}(\Theta)$ over all directions is unity, i.e.,

$$
\frac{1}{2} \int_{-1}^{1} a_{1}(\Theta) \mathrm{d} \cos \Theta=1
$$

In this description, $a_{1}(\Theta)$ is usually called the scattering or phase function. In this paper, we use

$$
P=\frac{\sqrt{Q^{2}+U^{2}}}{I}
$$

and when the Stokes parameter $U=0$, we use in addition

$$
P_{\mathrm{s}}=-\frac{Q}{I}
$$

which is positive when the vibrations perpendicular to the reference plane dominate the vibrations parallel to this plane. Both $P$ and $P_{\mathrm{s}}$ will be called the degree of linear polarization. For single scattering by particles with a scattering matrix like Eq. (1) and incident unpolarized light, the degree of linear polarization is given by

$$
P_{\mathrm{s}}(\Theta)=-\frac{b_{1}(\Theta)}{a_{1}(\Theta)}
$$

\section{Brief review of parameterized matrices for scattering in planetary atmospheres}

The 1,1-element of a scattering matrix as a function of scattering angle is often parameterized as a one-term Henyey-Greenstein function

$$
a_{1}(\Theta)=P_{\mathrm{HG}}(g, \Theta)=\frac{1-g^{2}}{\left(1+g^{2}-2 g \cos \Theta\right)^{3 / 2}} .
$$

Here, $-1<g<1$ is called the asymmetry parameter, and is equal to $\langle\cos \Theta\rangle=$ $\frac{1}{2} \int_{-1}^{1} a_{1}(\Theta) \cos \Theta \mathrm{d} \cos \Theta$. This function was introduced by Henyey and Greenstein [18] in a study of diffuse interstellar radiation. Van de Hulst [11] gives a review of its use in multiple light scattering studies. A common alternative parameterization employs a two-term HenyeyGreenstein function:

$$
a_{1}(\Theta)=f_{1} P_{\mathrm{HG}}\left(g_{1}, \Theta\right)+\left(1-f_{1}\right) P_{\mathrm{HG}}\left(g_{2}, \Theta\right)
$$

with $-1<g_{1}<1,-1<g_{2}<1$, and $0 \leq f_{1} \leq 1$. In the wake of Irvine [12], this function has been used extensively in multiple-scattering problems, especially when polarization was neglected.

In theoretical work on multiple scattering, Hovenier [19] used parameterized matrices as scattering matrices to test the adding/doubling method for polarized light. He used a two-term Henyey-Greenstein function for $a_{1}(\Theta)$, and further employed $a_{2}(\Theta)=a_{1}(\Theta), \quad b_{1}(\Theta)=$ $m \times \sin (n \Theta), a_{3}(\Theta)=\sqrt{a_{1}^{2}(\Theta)-b_{1}^{2}(\Theta)}$, and $b_{2}(\Theta) \equiv 0$ for certain values of the parameters $g_{1}, g_{2}, f_{1}$, 
$m$, and $n$. This choice for $b_{2}(\Theta)$ may be justified because this element affects scattering calculations only for second- and higher-order scattering if the incident light is unpolarized. Indeed, in a case study by Hansen [20] it was shown that neglecting the fourth row and column - the so-called $3 \times 3$ approximation - causes only marginal differences in the calculated brightness and linear polarization of atmospheres. If $b_{2}(\Theta) \equiv 0$, the Stokes parameter $V$ is decoupled from the Stokes parameters $I, Q$, and $U$, and the $3 \times 3$ approximation is exact for these three Stokes parameters.

In analyzing Pioneer 11 and Voyager 2 photopolarimetry of Saturn and its satellite Titan, various authors chose a parameterized matrix approach, sometimes combined with Mie theory. We will now give a brief review of the parameterized matrices used in these investigations. In all cases, the parameterized matrix had the structure given by Eq. (1) with $b_{2}(\Theta) \equiv 0$. Unless otherwise noted, $a_{1}(\Theta)$ was parameterized by a one- or two-term Henyey-Greenstein function.

As the degree of linear polarization as a function of phase angle for Titan hinted at particles with Rayleigh-like polarizing properties, Tomasko and Smith [13] and West et al. [14] chose to use a "factor times Rayleigh" approach for the 2,1-element, i.e.,

$$
-\frac{b_{1}(\Theta)}{a_{1}(\Theta)}=p \frac{\sin ^{2} \Theta}{1+\cos ^{2} \Theta}
$$

or, in other words, $P_{\mathrm{s}}(\Theta)$ is some parameter $p$ times the expression for $P_{\mathrm{s}}(\Theta)$ for Rayleigh scattering without depolarization (cf. Ref. [21]).

For the planet Saturn, Tomasko and Doose [22] chose $-b_{1}(\Theta) / a_{1}(\Theta)$ to be a piecewise linear function of $\Theta$, specified at $10^{\circ}$ increments of the scattering angle from $0^{\circ}$ to $180^{\circ}$. West et al. [23] used a similar formulation, but specified $-b_{1}(\Theta) / a_{1}(\Theta)$ at scattering angles corresponding to the phase angles at which Saturn was observed during the flyby of Voyager 2.

All studies discussed above except that of Hovenier [19] employed $a_{1}(\Theta)=a_{2}(\Theta)=a_{3}(\Theta)$. This choice is sometimes motivated by noting that, for spheres, $a_{2}(\Theta)=a_{1}(\Theta)$ and $a_{3}(\Theta)$ resembles $a_{1}(\Theta)$ for a large part of the scattering angle range [13,22]. For his studies of Titan's polarization, Stammes [15] also used $a_{1}(\Theta)=a_{2}(\Theta)$, but proposed an alternative description for $a_{3}(\Theta)$ :

$$
\frac{a_{3}(\Theta)}{a_{1}(\Theta)}=\frac{2 \cos \Theta}{1+\cos ^{2} \Theta}
$$

inspired by Rayleigh scattering without depolarization, for which this equation holds (cf. Ref. [21]).

Two other parameterizations are worth noting. First, Smith and Tomasko [24] used two-term Henyey-Greenstein phase functions for $a_{1}(\Theta)$ that were modified between the scattering angles of 80 and $140^{\circ}$, to inspect the Pioneer photometric data of Jupiter for evidence of spherical particles (which they did not find). Second, West et al. [23] used for Saturn, in addition to their approach outlined above, scattering matrices from Mie theory (pertaining to spherical particles), but they replaced the 1,2- and 2,1-elements with an expression based on Eq. (8).

\section{Requirements for parameterized scattering matrices}

This section describes requirements that a $4 \times 4$ real matrix should meet so that it cannot be physically excluded for not being a scattering matrix. A parameterized matrix that obeys all of 
these requirements will be referred to as a parameterized scattering matrix (PSM). Note that for a PSM it cannot be conclusively proven that a particle or collection of particles exists that has this PSM as its scattering matrix.

\subsection{Requirements at 0 and $180^{\circ}$ scattering angle}

In the exact forward and backward scattering directions, Eq. (1), which holds for randomly oriented particles that occur in equal numbers as their mirror particles, becomes even simpler $[25,26]$. In the exact forward direction $\left(\Theta=0^{\circ}\right)$, the following properties hold:

$$
\begin{aligned}
& a_{1} \geq 0, \\
& \left|a_{2}\right| \leq a_{1}, \\
& a_{3}=a_{2}, \\
& \left|a_{4}\right| \leq a_{1}, \\
& a_{4} \geq 2\left|a_{2}\right|-a_{1}, \\
& b_{1}=b_{2}=0 .
\end{aligned}
$$

In the exact backscattering direction $\left(\Theta=180^{\circ}\right)$, we have

$$
\begin{aligned}
& a_{1} \geq 0, \\
& 0 \leq a_{2} \leq a_{1}, \\
& a_{3}=-a_{2}, \\
& a_{4}=a_{1}-2 a_{2}, \\
& b_{1}=b_{2}=0 .
\end{aligned}
$$

It should be noted that most of the parameterizations described in Section 3 violate Eq. (18).

\subsection{The Cloude test}

The sharpest requirement for arbitrary scattering angles is based on the Cloude coherency matrix $\mathbf{T}$ of a given matrix $\mathbf{F}$ [27]. If all eigenvalues of $\mathbf{T}$ are nonnegative for a particular scattering angle, $\mathbf{F}$ can be a scattering matrix for that scattering angle. We refer to this requirement as the Cloude test. Note that for $\Theta=0$ and $180^{\circ}$, Eqs. (10)-(20) must still be satisfied.

For matrices satisfying Eq. (1), the Cloude coherency matrix is given by

$$
\begin{aligned}
& T_{11}=\frac{1}{2}\left(a_{1}+a_{2}+a_{3}+a_{4}\right), \\
& T_{22}=\frac{1}{2}\left(a_{1}+a_{2}-a_{3}-a_{4}\right), \\
& T_{33}=\frac{1}{2}\left(a_{1}-a_{2}+a_{3}-a_{4}\right), \\
& T_{44}=\frac{1}{2}\left(a_{1}-a_{2}-a_{3}+a_{4}\right), \\
& T_{12}=b_{1}-\mathrm{i} b_{2},
\end{aligned}
$$




$$
\begin{aligned}
& T_{21}=b_{1}+\mathrm{i} b_{2}, \\
& T_{i j}=0 \quad \text { otherwise, }
\end{aligned}
$$

where $\mathrm{i}=\sqrt{-1}$ and the argument $\Theta$ is omitted for brevity. We readily compute the eigenvalues of $\mathbf{T}$. The requirement that these eigenvalues must all be nonnegative then reduces to

$$
\begin{aligned}
& \left|a_{3}-a_{4}\right| \leq a_{1}-a_{2}, \\
& \sqrt{\left(a_{3}+a_{4}\right)^{2}+4 b_{1}^{2}+4 b_{2}^{2}} \leq a_{1}+a_{2},
\end{aligned}
$$

which agrees with Eq. (10) of Ref. [16]. A candidate PSM will be called a PSM if it passes the Cloude test for all scattering angles and satisfies the requirements at the scattering angles of 0 and $180^{\circ}$ as specified by Eqs. (10)-(20). Thus not every parameterized matrix is a PSM.

Even if $b_{2}(\Theta) \equiv 0$ is chosen, $a_{4}(\Theta)$ cannot just be ignored on constructing a PSM. Indeed, for any given $a_{1}(\Theta), a_{2}(\Theta), a_{3}(\Theta)$, and $b_{1}(\Theta)$, we should require for a PSM that there exists at least one function $a_{4}(\Theta)$ so that Eqs. (28) and (29) are satisfied for all scattering angles, as well as Eqs. (10) $-(15)$ and (16) $-(20)$ at $\Theta=0$ and $180^{\circ}$, respectively.

We note that a matrix that passes the Cloude test automatically satisfies

$$
a_{1}(\Theta) \geq 0
$$

and

$$
\begin{array}{ll}
a_{1}(\Theta) \geq\left|a_{i}(\Theta)\right|, & i=2,3,4, \\
a_{1}(\Theta) \geq\left|b_{j}(\Theta)\right|, & j=1,2 .
\end{array}
$$

As an example of what may happen if the Cloude test is not fulfilled we consider the following matrix, where we used Eq. (8) and $a_{1}(\Theta)=a_{2}(\Theta)=a_{3}(\Theta)$,

$$
\mathbf{F}\left(90^{\circ}\right)=a_{1}\left(90^{\circ}\right)\left[\begin{array}{cccc}
1 & -p & 0 & 0 \\
-p & 1 & 0 & 0 \\
0 & 0 & 1 & 0 \\
0 & 0 & 0 & \frac{a_{4}\left(90^{\circ}\right)}{a_{1}\left(90^{\circ}\right)}
\end{array}\right]
$$

with $p \neq 0$. This matrix does not pass the Cloude test, regardless of the value of the 4,4-element. Now consider a scattering event where the Stokes vector of the incident light is given by $[1,0,1,0]$. Then the Stokes vector of the scattered light is $[1,-p, 1,0]$ which is not allowed since it does not satisfy $\sqrt{Q^{2}+U^{2}+V^{2}} \leq I$.

\section{Expansion of scattering matrices in generalized spherical functions}

It is often convenient to expand the elements of a scattering matrix in series of generalized spherical functions of the scattering angle. Such an expansion is especially handy in multiplescattering calculations (see, e.g., Ref. [28]). Also, from a table of expansion coefficients it is easy to retrieve the scattering matrix at arbitrary scattering angles, with an accuracy determined by the 
truncation of the series and the accuracy of the expansion coefficients. It stands to reason that an expansion is especially useful if the expansion coefficients fall off quickly with increasing degree.

The generalized spherical functions $P_{m, n}^{l}(x)$ are related to the Jacobi polynomials $P_{s}^{\alpha, \beta}(x)$ as follows (cf. Ref. [6]):

$$
P_{m, n}^{l}(x)=\frac{(-\mathrm{i})^{\alpha}}{2^{(\alpha+\beta) / 2}} \sqrt{\frac{s !(s+\alpha+\beta) !}{(s+\alpha) !(s+\beta) !}}(1-x)^{\alpha / 2}(1+x)^{\beta / 2} P_{s}^{\alpha, \beta}(x),
$$

where $\alpha=|n-m|, \beta=|n+m|$, and $s=l-\max (|m|,|n|)$.

The expansion of the scattering matrix in generalized spherical functions is given by [29]

$$
\begin{aligned}
& a_{1}(\Theta)=\sum_{l=0}^{\infty} \alpha_{1}^{l} P_{0,0}^{l}(\cos \Theta), \\
& a_{2}(\Theta)+a_{3}(\Theta)=\sum_{l=2}^{\infty}\left(\alpha_{2}^{l}+\alpha_{3}^{l}\right) P_{2,2}^{l}(\cos \Theta), \\
& a_{2}(\Theta)-a_{3}(\Theta)=\sum_{l=2}^{\infty}\left(\alpha_{2}^{l}-\alpha_{3}^{l}\right) P_{2,-2}^{l}(\cos \Theta), \\
& a_{4}(\Theta)=\sum_{l=0}^{\infty} \alpha_{4}^{l} P_{0,0}^{l}(\cos \Theta), \\
& b_{1}(\Theta)=\sum_{l=2}^{\infty} \beta_{1}^{l} P_{0,2}^{l}(\cos \Theta), \\
& b_{2}(\Theta)=\sum_{l=2}^{\infty} \beta_{2}^{l} P_{0,2}^{l}(\cos \Theta) .
\end{aligned}
$$

The expansion coefficient $\alpha_{1}^{l}$ is given by [29]

$$
\alpha_{1}^{l}=\left(l+\frac{1}{2}\right) \int_{-1}^{+1} P_{0,0}^{l}(\cos \Theta) a_{1}(\Theta) \mathrm{d} \cos \Theta .
$$

The other expansion coefficients, $\alpha_{2}^{l} \pm \alpha_{3}^{l}, \alpha_{4}^{l}, \beta_{1}^{l}$, and $\beta_{2}^{l}$ are obtained from similar integrals.

Now, combining Eq. (34) and an expansion theorem for Jacobi polynomials due to Szegö (Theorem 9.1.1 of Ref. [30]), it can be shown that any of the expansions given in Eqs. (35)-(40) converges pointwise for every value of $\cos \Theta$ on the closed interval $[-1,+1]$ and has exponentially decreasing expansion coefficients if the corresponding function from the set

$$
a_{1}(\Theta), \quad \frac{a_{2}(\Theta)+a_{3}(\Theta)}{(1+\cos \Theta)^{2}}, \quad \frac{a_{2}(\Theta)-a_{3}(\Theta)}{(1-\cos \Theta)^{2}}, \quad a_{4}(\Theta), \quad \frac{b_{1}(\Theta)}{1-\cos ^{2} \Theta} \quad \text { and } \frac{b_{2}(\Theta)}{1-\cos ^{2} \Theta}
$$

is analytic in $\cos \Theta$ in the interior of an ellipse in the complex plane with foci at \pm 1 . Here, " $\alpha_{1}^{l}$ decreases exponentially" means that $\alpha_{1}^{l}$ decreases with increasing $l$ in the sense that

$$
\liminf _{l \rightarrow \infty}\left|\alpha_{1}^{l}\right|^{-1 / l}=R
$$

for some constant $R$ exceeding 1 , and similarly for the expansion coefficients $\alpha_{2}^{l} \pm \alpha_{3}^{l}, \alpha_{4}^{l}, \beta_{1}^{l}$, and $\beta_{2}^{l}$. Szegö's theorem further states that $R$ is equal to the sum of the semi-axes of the largest ellipse of 
convergence with foci at \pm 1 . It should be noted that if both $\alpha_{2}^{l}+\alpha_{3}^{l}$ and $\alpha_{2}^{l}-\alpha_{3}^{l}$ decrease exponentially, the same is true for $\alpha_{2}^{l}$ and $\alpha_{3}^{l}$ separately, and vice versa.

\section{Parameterization for common types of particles}

In this section we present parameterizations that can mimic a broad range of scattering matrices as functions of scattering angle using a few parameters. As an example, this section will give a parameterization that may be used to simulate the single-scattering behavior of particles that are not large compared to the wavelength, particles in the Rayleigh-Gans domain, or a variety of irregularly shaped particles at visible wavelengths. A particle is considered to be in the Rayleigh-Gans domain if its refractive index is such that both $|m-1| \ll 1$ and $\pi d|m-1| \ll \lambda$, where $m$ and $d$ are the complex refractive index and a characteristic linear dimension of the particle, respectively, and $\lambda$ is the wavelength [5]. In Sections 6.2 and 6.3 these parameterizations are judged in light of Sections 4 and 5.

\subsection{Sample parameterizations}

Mishchenko et al. [31] give a comprehensive overview of measurements and calculations of light scattering by particles covering a great variety of sizes, shapes, and compositions. This book chapter provides many useful references to papers dealing with scattering by randomly oriented mineral particles in the visible part of the spectrum. The scattering matrices reported in these papers exhibit many common features:

- Phase functions $a_{1}(\Theta)$ with pronounced maxima at $\Theta=0^{\circ}$.

- Ratios $a_{2}(\Theta) / a_{1}(\Theta)$ that generally decrease from approximately 1 at $\Theta=0^{\circ}$ to a value between 0 and 1 at $\Theta=180^{\circ}$.

- Ratios $a_{3}(\Theta) / a_{1}(\Theta)$ that decrease from approximately 1 at $\Theta=0^{\circ}$ to $-a_{2}(\Theta) / a_{1}(\Theta)$ at $\Theta=180^{\circ}$, and have zeros near or beyond $\Theta=90^{\circ}$.

- Ratios $a_{4}(\Theta) / a_{1}(\Theta)$ that decrease from approximately 1 at $\Theta=0^{\circ}$ to approximately $1-2 a_{2}(\Theta) / a_{1}(\Theta)$ at $\Theta=180^{\circ}$, and have zeros near or beyond $\Theta=90^{\circ}$.

- Ratios $-b_{1}(\Theta) / a_{1}(\Theta)$ that are zero at $\Theta=0^{\circ}$ and $\Theta=180^{\circ}$, and have a maximum near or beyond $\Theta=90^{\circ}$. This holds especially for particles that are not large compared to the wavelength, for particles in the Rayleigh-Gans domain, and for a variety of irregularly shaped particles.

- Ratios $b_{2}(\Theta) / a_{1}(\Theta)$ that are often small.

On the basis of these papers we now define the following class of candidate PSM's:

$$
\begin{aligned}
& a_{1}(\Theta)=f_{1} P_{\mathrm{HG}}\left(g_{1}, \Theta\right)+\left(1-f_{1}\right) P_{\mathrm{HG}}\left(g_{2}, \Theta\right), \\
& \frac{a_{2}(\Theta)}{a_{1}(\Theta)}=h+(1-h) \cos \Theta, \\
& \frac{a_{3}(\Theta)}{a_{2}(\Theta)}=\frac{2 \cos \Theta}{1+\cos ^{2} \Theta},
\end{aligned}
$$




$$
\begin{aligned}
& -\frac{b_{1}(\Theta)}{a_{1}(\Theta)}=p \frac{\sin ^{2} \Theta}{1+\cos ^{2} \Theta}, \\
& \frac{b_{2}(\Theta)}{a_{1}(\Theta)} \equiv 0, \\
& a_{4}(\Theta) \text { is not specified, }
\end{aligned}
$$

with the five dimensionless parameters $g_{1}, g_{2}, f_{1}, h$, and $p$.

Eq. (45) provides a generalization of the parameterizations that assume $a_{2}(\Theta)=a_{1}(\Theta)$ (cf. Section 3). The choice for $a_{3}(\Theta)$ given in Eq. (46) is similar to the choice that Stammes [15] made, but with $a_{1}(\Theta)$ replaced by $a_{2}(\Theta)$, in order to assure $a_{3}\left(180^{\circ}\right)=-a_{2}\left(180^{\circ}\right)$ (cf. Eq. (18)). Eq. (46) holds exactly for Rayleigh scattering. Parameters $h$ and $p$ equal $a_{2}\left(90^{\circ}\right) / a_{1}\left(90^{\circ}\right)$ and $-b_{1}\left(90^{\circ}\right) / a_{1}\left(90^{\circ}\right)$, respectively.

\subsection{Constraints for the parameter values}

For the parameters $g_{1}, g_{2}$, and $f_{1}$, we use the conventional constraints for the two-term Henyey-Greenstein function, i.e., $-1<g_{1}<1,-1<g_{2}<1$, and $0 \leq f_{1} \leq 1$ (cf. Section 3).

The parameterization given in Eqs. (44)-(49) then obeys the forward- and backward-scattering requirements as expressed by Eqs. (10)-(12), (15)-(16), (18) and (20). In addition, Eq. (17) is satisfied if $0.5 \leq h \leq 1$ and then a function $a_{4}(\Theta)$ can be constructed so that Eqs. (13), (14), and (19) are also satisfied.

Limits on the fifth parameter, $p$, may be obtained from the Cloude test in the following way. We will first consider the case $h=1$. Regardless of the choice for $a_{3}(\Theta)$, we get from Eq. $(28) a_{4}(\Theta)=$ $a_{3}(\Theta)$, and from Eq. (29) $b_{1}^{2}(\Theta) \leq a_{1}^{2}(\Theta)-a_{3}^{2}(\Theta)$. Using Eq. (46), this yields after some algebra

$$
p^{2} \leq 1
$$

In other words, this candidate PSM passes the Cloude test for all scattering angles if $|p| \leq 1$.

When $h<1$, the algebra gets overly complicated, but we will consider the special cases $\Theta=0$, 90, and $180^{\circ}$. For $\Theta=0^{\circ}$, we have $a_{1}=a_{2}=a_{3}$, and $b_{1}=0$. This satisfies Eqs. (28) and (29) if also $a_{4}=a_{1}$, which is allowed by Eqs. (13) and (14). For $\Theta=180^{\circ}$, we have $a_{2}<a_{1}, a_{3}=-a_{2}$, and $b_{1}=0$. This matrix satisfies Eqs. (28) and (29) for a set of $a_{4}$ 's, including $a_{4}=a_{1}-2 a_{2}$, as required by Eq. (19). However, for $\Theta=90^{\circ}, a_{2} / a_{1}=h, a_{3} / a_{1}=0$, and $b_{1} / a_{1}=-p$, yielding from Eq. (29),

$$
4 p^{2} \leq(1+h)^{2}-\left(\frac{a_{4}}{a_{1}}\right)^{2} .
$$

Since, according to Eq. (28), $a_{4}$ is allowed to be zero, this sets a constraint, $|p| \leq(1+h) / 2$. Thus, for $h<1$, the interval of valid values for $p$ is smaller than for $h=1$. Further inspection shows that when the upper limit is taken for $|p|$, the test is passed for all scattering angles up to $90^{\circ}$, and most of the scattering angles larger than that, but not for scattering angles slightly larger than $90^{\circ}$. However, if $|p|$ is taken a little smaller, the candidate PSM passes the Cloude test for any $\Theta$. For instance, when $h=0.95, p= \pm 0.97$ still works.

Whenever parameterized matrices are used as scattering matrices, (a) the requirements in the forward- and backward-scattering directions (Eqs. (10)-(20)) should be checked, and (b) the Cloude test, (Eqs. (28) and (29)) should be performed analytically for all scattering angles, or numerically for 

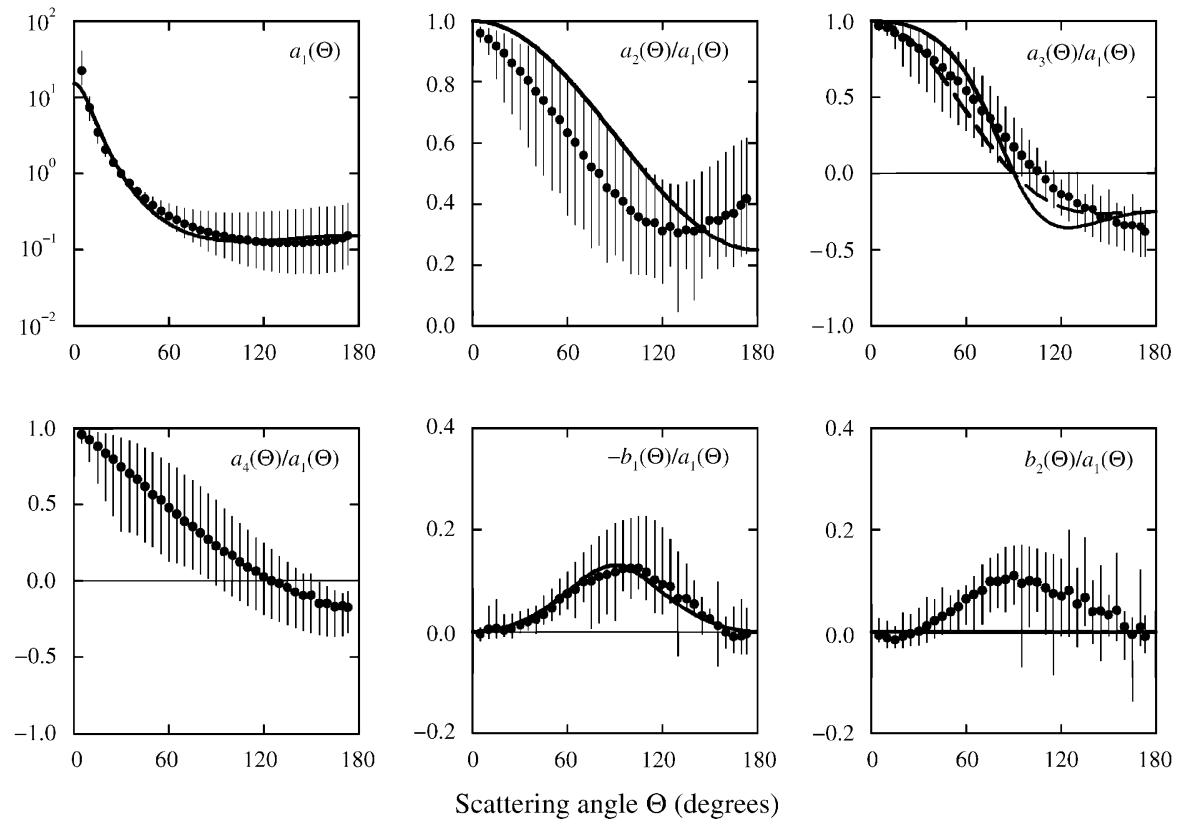

Fig. 2. Dots: the elements of the scattering matrix as functions of scattering angle of randomly oriented aerosols measured in the laboratory by Volten et al. [34], averaged over seven types of mineral aerosols and two wavelengths (441.6 and $632.8 \mathrm{~nm}$ ). The upright bars indicate the domains covered by all measurements, i.e., the area between the best minimum and maximum values measured for each angle. Thick solid lines: a PSM with $g_{1}=0.8, g_{2}=-0.15$, $f_{1}=0.8, p=0.13, h=0.625$, and $a_{3}(\Theta) / a_{2}(\Theta)=2 \cos \Theta /\left(1+\cos ^{2} \Theta\right)$. Thick dashed line: another PSM with the same parameters, but with $a_{3}(\Theta) / a_{2}(\Theta)=\cos \Theta$. Note that the parameterized functions $b_{2}(\Theta) / a_{1}(\Theta)$ coincide with the $\Theta$-axis, and that the parameterized functions $a_{4}(\Theta) / a_{1}(\Theta)$ are absent (cf. Eqs. (48) and (49)).

a large set of distinct scattering angles. One should be aware that the ranges derived here are theoretical. For instance, particles are generally forward scattering and often positively polarizing for most of the scattering angle range, unless extraordinary conditions are imposed $[32,33]$. So, one should be wary of negative values of $f_{1} g_{1}+\left(1-f_{1}\right) g_{2}$ and $p$.

Fig. 2 shows results of laboratory measurements by Volten et al. [34], compared with a particular realization of Eqs. (44)-(49) that gives a fair representation. Also shown is an alternative parameterization for the 3,3-element,

$$
\frac{a_{3}(\Theta)}{a_{2}(\Theta)}=\cos \Theta
$$

that follows more closely the behavior that is sometimes observed in measurements. Note that in either case $a_{3}\left(90^{\circ}\right)=0$. We have verified that both parameterized matrices are PSMs.

\subsection{Expansions in generalized spherical functions}

We will consider here three parameterized matrices, labeled P1, P2, and P3. P1 is characterized by Eqs. (44)-(49) with $g_{1}=0.8, g_{2}=-0.7, f_{1}=0.938$, and $p=0.9$, which are values found for 

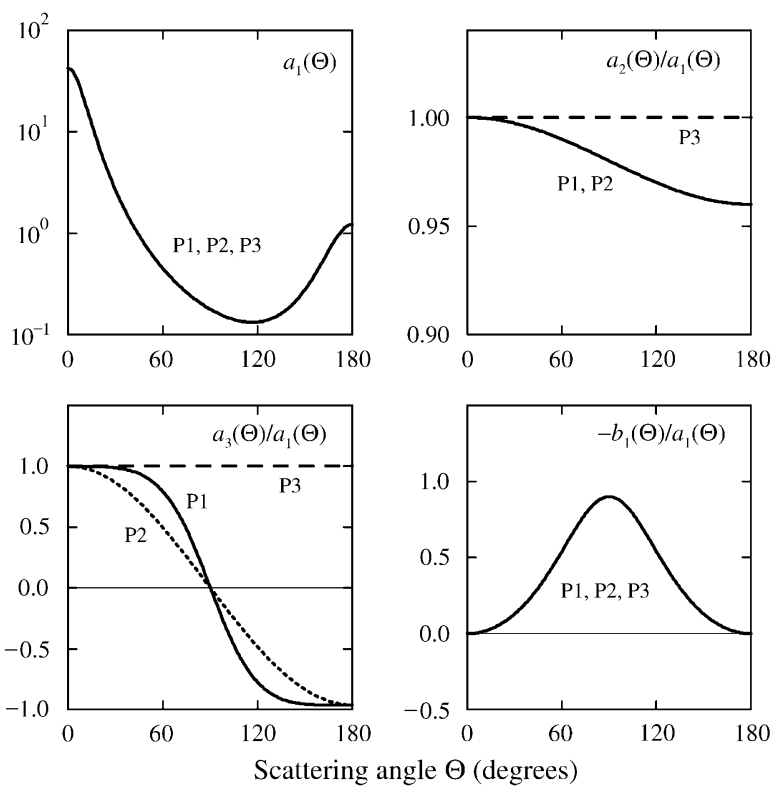

Fig. 3. The elements of the three parameterized matrices described in Section 6.3, as functions of scattering angle. The corresponding coefficients for the expansions in generalized spherical functions are shown in Fig. 4.

a portion of Jupiter's atmosphere in red wavelengths [24]. Also, we chose $h=0.96$ arbitrarily. For $\mathrm{P} 2$, the same parameter values were used, but with the 3,3-element given by Eq. (52). The third parameterization is a more crude one that has the same $a_{1}(\Theta)$ and $b_{1}(\Theta)$ as P1 and P2, but has $a_{1}(\Theta)=a_{2}(\Theta)=a_{3}(\Theta)$. P1 and P2 are PSMs since for either of these matrices, a function $a_{4}(\Theta)$ exists so that the Cloude test is passed for all scattering angles and Eqs. (10)-(20) are satisfied. P3 is not a PSM (cf. Eq. (33)). The elements of the parameterized matrices as functions of scattering angle are shown in Fig. 3.

Fig. 4 shows the behavior of the expansion coefficients $\alpha_{1}^{l}, \alpha_{2}^{l}, \alpha_{3}^{l}$, and $\beta_{1}^{l}$ for these parameterized matrices. To compute the integrals like Eq. (41), we have used Gauss-Legendre integration with 3000 Gaussian points in $\cos \Theta$. Since $a_{1}(\Theta)$ and $b_{1}(\Theta)$ are the same for these parameterizations, there is only one curve labeled $\alpha_{1}^{l}$ and one labeled $\beta_{1}^{l}$. Both tend to straight lines in this logarithmic plot, signifying an exponential decrease of the expansion coefficients with $l$ for both elements. For P1, $\alpha_{2}^{l}$ and $\alpha_{3}^{l}$ are always positive and within a line thickness equal to $\alpha_{1}^{l}$. For P2, $\alpha_{3}^{l}$ is negative for $l \geq 43$ but for larger $l$, its absolute value tends to that of $\alpha_{2}^{l}$. For P3, $\alpha_{2}^{l}=\alpha_{3}^{l}$ for all $l$. Here, $\alpha_{2}^{l}$ and $\alpha_{3}^{l}$ are negative for odd $l, l \geq 27$. For P2 and P3, $\left|\alpha_{2}^{l}\right|$ and $\left|\alpha_{3}^{l}\right|$ do not decrease exponentially in Fig. 4.

Using the expansion theorem discussed in Section 5, we can explain the way in which the coefficients decrease with $l$ as follows. Starting with $\alpha_{1}^{l}$, the Henyey-Greenstein function $P_{\mathrm{HG}}(g, \Theta)$ (Eq. (6)) is analytic in $\cos \Theta$ on the entire complex plane, except for the pole $\cos \Theta=\frac{1}{2}\left(g+g^{-1}\right)$. Therefore, for $|g|<1$, an ellipse with foci at $\cos \Theta= \pm 1$ exists in the interior of which $P_{\mathrm{HG}}(g, \Theta)$ is analytic in $\cos \Theta$, so that the expansion of $P_{\mathrm{HG}}(g, \Theta)$ in generalized spherical functions converges with exponentially decreasing coefficients. Further, for decreasing $g$, the ellipse of convergence increases in size, since $R=|g|^{-1}$, and the coefficients $\alpha_{1}^{l}$ fall off more rapidly. This is a well-known 


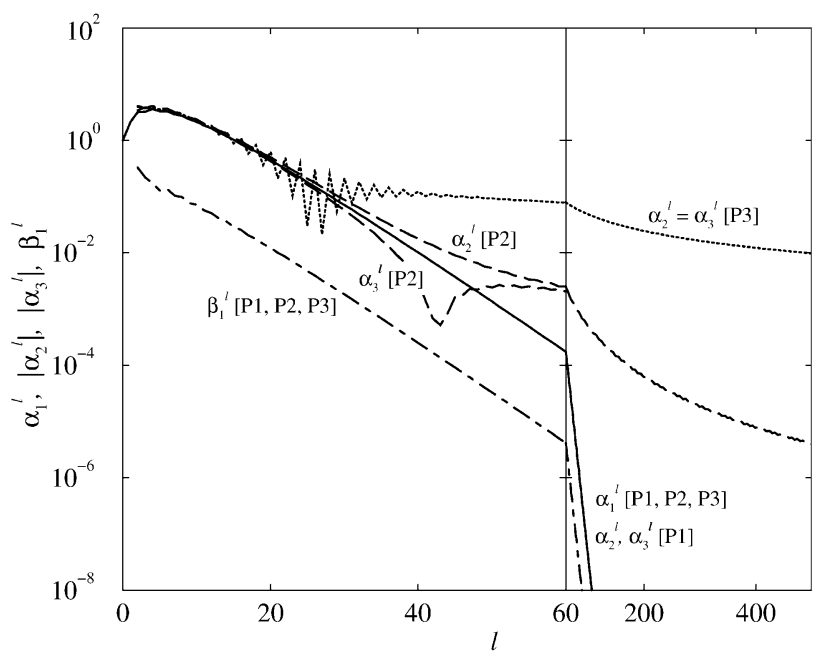

Fig. 4. Expansion coefficients $\alpha_{1}^{l},\left|\alpha_{2}^{l}\right|,\left|\alpha_{3}^{l}\right|$, and $\beta_{1}^{l}$ as functions of $l$ for the parameterized matrices that are shown in Fig. 3. The right panel is a continuation of the left one, but with a different scale. Solid line: $\alpha_{1}^{l}$ for all models. Dot-dashed: $\beta_{1}^{l}$ for all models. Dashed: $\left|\alpha_{2}^{l}\right|$ and $\left|\alpha_{3}^{l}\right|$ for P2. Dotted: $\left|\alpha_{2}^{l}\right|$ and $\left|\alpha_{3}^{l}\right|$ for P3. For P1, the curves for $\left|\alpha_{2}^{l}\right|$ and $\left|\alpha_{3}^{l}\right|$ coincide within the line thickness with the curve for $\alpha_{1}^{l}$.

Table 1

Poles (values of $\cos \Theta$ in the complex plane) for the expressions in Eq. (42) relevant to parameterized matrices P1, P2, and $\mathrm{P} 3 . R$ is the sum of the semi-axes of the largest ellipse of convergence with foci at \pm 1 for an expansion in generalized spherical functions

\begin{tabular}{llll}
\hline Coefficient & Expression in Eq. (42) & Poles & $R$ \\
\hline$\alpha_{1}^{l}$ & $a_{1}(\Theta)$ & $\frac{1+g_{1}^{2}}{2 g_{1}}, \frac{1+g_{2}^{2}}{2 g_{2}}$ & $\min \left(\frac{1}{g_{1}}, \frac{1}{g_{2}}\right)$ \\
$\alpha_{2}^{l} \pm \alpha_{3}^{l}$ & $\frac{a_{2}(\Theta) \pm a_{3}(\Theta)}{(1 \pm \cos \Theta)^{2}}$ & for P1: $\frac{1+g_{1}^{2}}{2 g_{1}}, \frac{1+g_{2}^{2}}{2 g_{2}}, \pm \mathrm{i}$ & $\min \left(\frac{1}{g_{1}}, \frac{1}{g_{2}}, 1+\sqrt{2}\right)$ \\
& for P2: $\frac{1+g_{1}^{2}}{2 g_{1}}, \frac{1+g_{2}^{2}}{2 g_{2}}, \mp 1$ & No ellipse \\
$\beta_{1}^{l}$ & for P3: $\frac{1+g_{1}^{2}}{2 g_{1}}, \frac{1+g_{2}^{2}}{2 g_{2}}, \mp 1$ & No ellipse \\
\hline
\end{tabular}

result, as for $g \neq 0$

$$
\alpha_{1}^{l}=(2 l+1) g^{l}
$$

and for $g=0, P_{\mathrm{HG}}(0, \Theta) \equiv 1[11]$. For a two-term Henyey-Greenstein phase function, the rate at which $\alpha_{1}^{l}$ decreases is determined by the larger of $\left|g_{1}\right|$ and $\left|g_{2}\right|$.

Table 1 lists the poles (values of $\cos \Theta$ in the complex plane) and the values of $R$ for all expansion coefficients relevant to our parameterizations. For P2 and P3, there is no ellipse of convergence for 
$\alpha_{2}^{l} \pm \alpha_{3}^{l}$ with foci in $\cos \Theta= \pm 1$. The rate at which $\alpha_{2}^{l}$ and $\alpha_{3}^{l}$ (for P1) and $\beta_{1}^{l}$ (for all parameterizations) decrease is determined by the poles of $a_{1}(\Theta)$, since for this function, both $\left|g_{1}\right|$ and $\left|g_{2}\right|$ are larger than $1 /(\sqrt{2}+1)$.

The results in Fig. 4 do not imply that only PSMs with $a_{3}(\Theta) / a_{2}(\Theta)=2 \cos \Theta /\left(1+\cos ^{2} \Theta\right)$ are practical. When only a modest accuracy is required (e.g. due to measurement errors), other shapes for the scattering angle dependence of the scattering matrix elements may work well enough even though the expansion coefficients may not fall off exponentially.

\section{Parameterized scattering matrices in atmospheric analysis}

\subsection{Models and method}

This section aims to illustrate the applicability of PSMs for interpreting observations of the brightness and polarization of a planetary atmosphere. In a first case study, the scattering matrix of a collection of aggregated particles was computed. By performing multiple-scattering calculations using this matrix, we simulated measurements of the brightness and state of polarization of the light reflected by a model atmosphere. Subsequently, a PSM was derived that reproduced these measurements as closely as possible. This PSM was then compared with the original scattering matrix. In a second case study, this process was repeated for a collection of spheroidal particles.

The properties (especially the size) of the particles in the two case studies were chosen so that $-b_{1}(\Theta) / a_{1}(\Theta)$ is Rayleigh-like, that is, showing a large maximum at a scattering angle near $90^{\circ}$. Both cases involve nonspherical particles, giving rise to an $a_{2}(\Theta)$ that is different from $a_{1}(\Theta)$.

Step 1. For the first case study, we considered four nonabsorbing aggregated particles each consisting of eight touching identical spherical monomers. Each of the monomers had a size parameter (ratio of circumference to wavelength) of 1.5. The size parameter of the sphere that has the same volume as each aggregate is 3 . The refractive index was chosen to be $1.33+0 \mathrm{i}$. A ballistic particle-cluster aggregation procedure, as described in Ref. [35], was used to form the aggregates. Although this aggregation formalism does not take restructuring of the growing aggregate into account, the resulting particles resemble aggregates observed in the laboratory [36,37]. The scattering matrices for these particles were calculated using the versatile discrete-dipole approximation as implemented in version $5 \mathrm{a} 9$ of the computer program DDSCAT [38,39]. Here, we averaged over $9^{3}$ orientations. Fig. 5 shows the four aggregates in the discrete-dipole representation used. The four scattering matrices were subsequently averaged using the respective scattering cross sections as weights, assuming that each of the four configurations occurs in equal numbers. The resulting matrix was normalized so that Eq. (2) holds. Since we know that repeating this procedure for the mirror particles would yield a similar scattering matrix, but with the signs of the 1,3-, 1,4-, 2,3-, 2,4-, 3,1-, 3,2-, 4,1-, and 4,2-elements reversed, we chose these elements to be zero. Therefore, we could treat this matrix as the scattering matrix of a mixture of randomly oriented particles and their mirror particles in equal numbers. The elements of the resulting scattering matrix as functions of scattering angle are shown as solid curves in Fig. 6. Within the numerical accuracy, this matrix satisfies Eqs. (10)-(20) and passes the Cloude test for all scattering angles. 

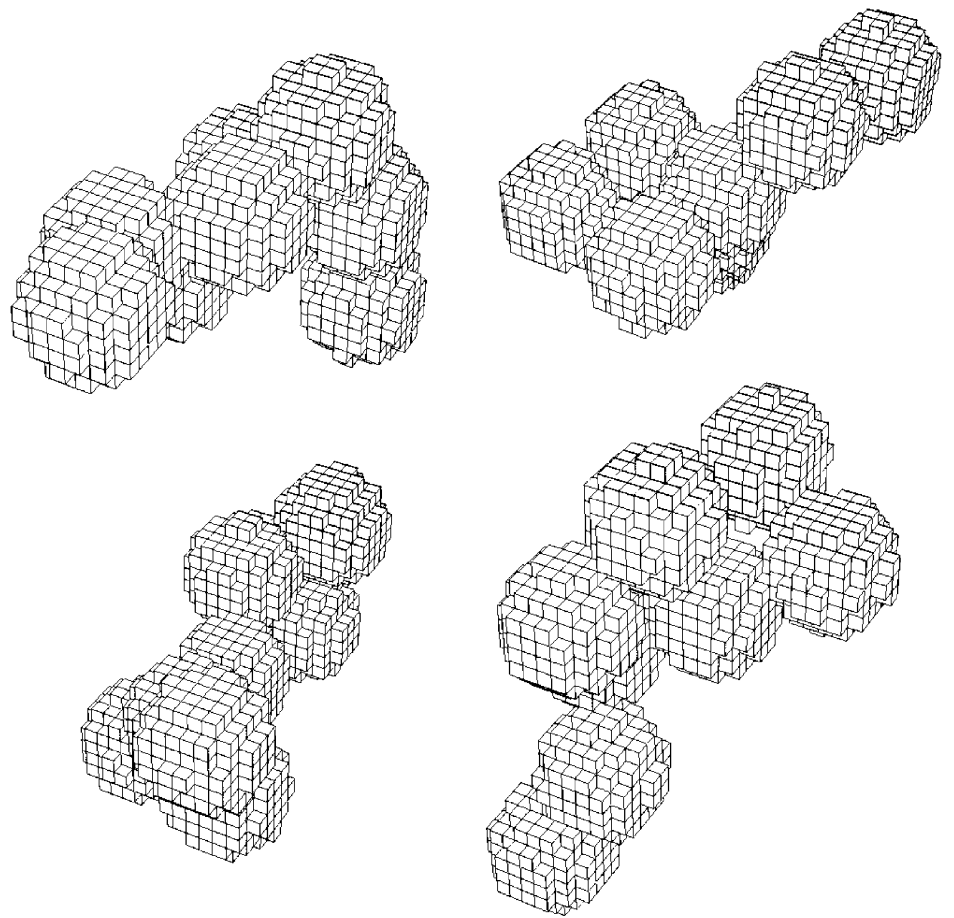

Fig. 5. Visualization of the four aggregates used in the first case study. Each aggregate was generated according to a ballistic particle-cluster aggregation procedure and consists of eight monomers that each had a size parameter of 1.5 . The midpoints of the cubes correspond to the locations of the dipoles in the discrete-dipole approximation. The inter-dipole distance, $d$, was chosen so that $2 \pi|m| d / \lambda=0.5$, where $m$ is the refractive index $(1.33+0 \mathrm{i})$ and $\lambda$ is the wavelength.

For the second case study, we considered a collection of oblate spheroidal particles with a refractive index of $1.33+0 \mathrm{i}$ and an aspect ratio of 3 . The size distribution of the equal-volume spheres was chosen to be a gamma-distribution [21] corresponding to an effective size parameter of 3 and an effective variance of 0.1 . The scattering matrix was calculated with the highly efficient $T$-matrix method as implemented in the double-precision $T$-matrix code of M.I. Mishchenko (version 14 April 1997) [40,41], using analytical averaging over particle orientations. The elements of the calculated scattering matrix as functions of scattering angle are shown as solid curves in Fig. 7. This matrix satisfies Eqs. (10)-(20), passes the Cloude test for all scattering angles, and is normalized so that Eq. (2) holds.

We will refer to these calculated scattering matrices and their elements as the original scattering matrices and elements.

Step 2. Next, for each case study, we considered a homogeneous, plane-parallel atmosphere with an optical thickness of 1 , and bounded below by a completely absorbing surface. The scattering matrices for volume elements of these atmospheres are the original matrices calculated during the previous step. Note that the albedo for single scattering equaled 1 , since the imaginary parts of the refractive indices of the particles equaled zero.

Assuming a distant light source above the atmospheres, we employed the full vectorial description of the adding/doubling method [19,28,42], using expansions of the scattering matrices in 

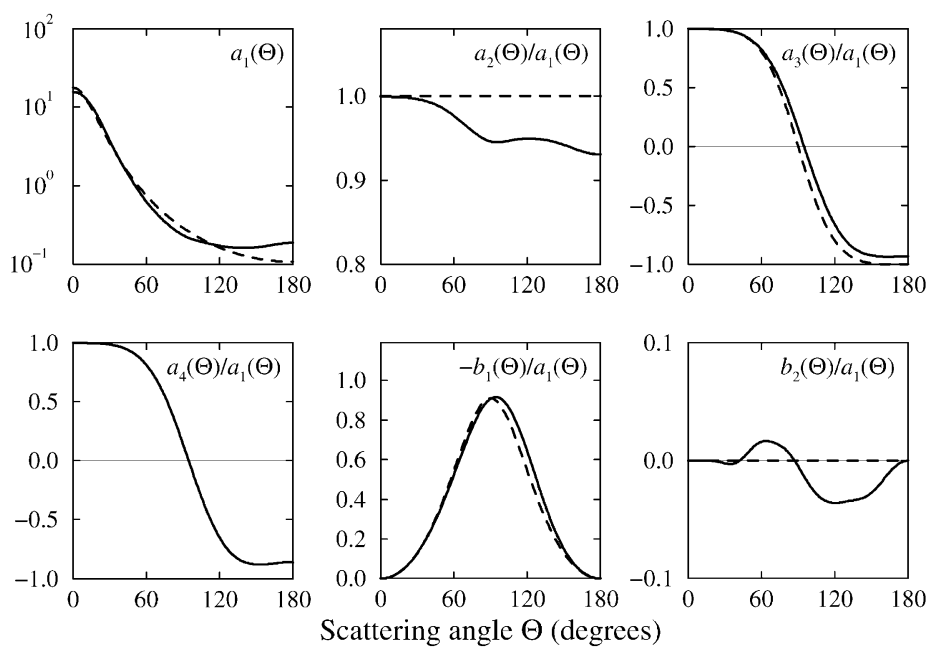

Fig. 6. Solid: the elements of the original scattering matrix used in the first case study as functions of scattering angle. This matrix is the average (weighted with the scattering cross sections) of the scattering matrices of the four particles shown in Fig. 5. Each of these four matrices itself is the average over $9^{3}$ orientations. Dashed: the elements of the parameterized scattering matrix that were retrieved from simulated measurements based on the original matrix.
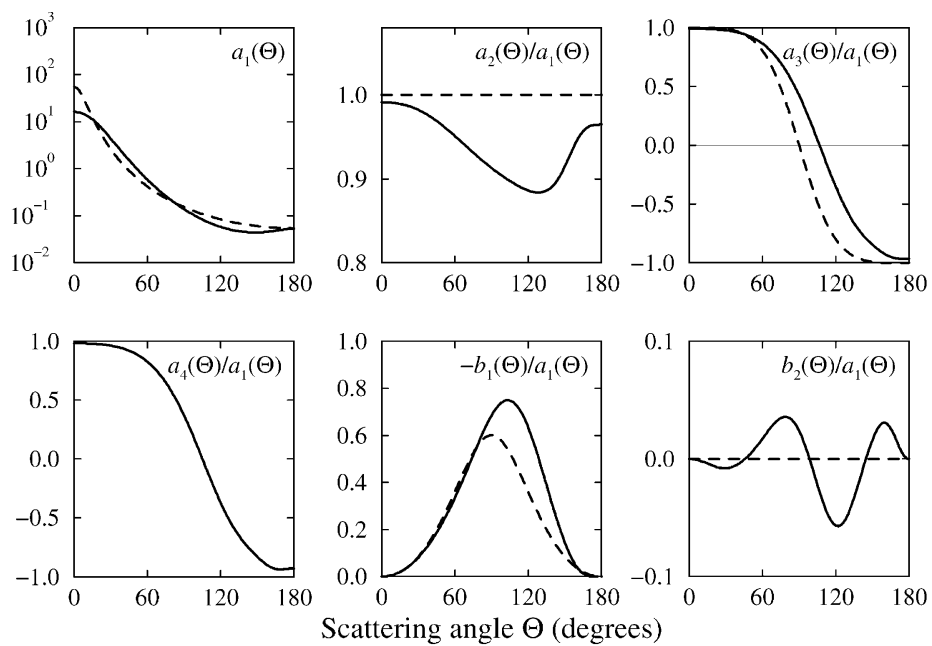

Fig. 7. Solid: the elements of the original scattering matrix used in the second case study as functions of scattering angle. These were calculated for an ensemble of randomly oriented oblate spheroids with a refractive index of $1.33+0 \mathrm{i}$ and an aspect ratio of 3 . The volume-equivalent size distribution corresponded to a gamma-distribution with an effective size parameter of 3 and an effective variance of 0.1. Dashed: the elements of the parameterized scattering matrix that were retrieved from simulated measurements based on the original matrix.

generalized spherical functions. In this manner, all four Stokes parameters of the light emerging from the top of the atmospheres were calculated for a number of viewing geometries. These geometries are characterized by $\mu_{0}=-\cos \theta_{0}, \mu=\cos \theta$, and $\phi-\phi_{0}$, where $\theta_{0}$ and $\theta$ are the zenith angles of the incident and scattered light, respectively, and $\phi_{0}$ and $\phi$ are the corresponding 
azimuthal angles, measured clockwise when looking up. For both case studies, values of 0.2-1.0 were considered for both $\mu$ and $\mu_{0}$, with steps of 0.1 ; for $\phi-\phi_{0}$, values of $0-180^{\circ}$ with steps of $30^{\circ}$ were considered. This adds up to a total of 567 geometries. The incident light was unpolarized, and its flux per unit area perpendicular to the incident beam equaled $\pi$. The calculated brightness and degree of linear polarization were treated as simulated measurements.

Step 3. We subsequently reproduced these simulated measurements as closely as possible using the parameterization given by Eqs. (44)-(49). We restricted ourselves to a one-term HenyeyGreenstein function for the 1,1-element. The homogeneous, plane-parallel model atmosphere again had an optical thickness of 1 , was bounded below by a completely absorbing surface, and was illuminated from above by the same light source as in Step 2. The albedo for single scattering equaled 1.

Initial values were chosen for the free parameters $g_{1}, p$, and $h$ to obtain a PSM to start with. We chose to fit the brightness and the degree of linear polarization alternately. First, fixing $p$ and $h$, the remaining parameter $g_{1}$ was varied so that it minimized the function

$$
\Delta I\left(g_{1}\right)= \begin{cases}\sum_{i=1}^{567}\left[I_{\mathrm{c}, i}\left(g_{1}\right) / I_{\mathrm{m}, i}-1\right]^{2}, & 0.3 \leq g_{1} \leq 0.95 \\ \Delta I_{\text {large }} & \text { otherwise }\end{cases}
$$

where $i$ numbers the geometries, and $I_{\mathrm{c}, i}$ and $I_{\mathrm{m}, i}$ are the calculated and "measured" brightnesses for the geometry $i$, respectively. $\Delta I$ was assigned a large value, $\Delta I_{\text {large }}$, for $g_{1}<0.3$ and $g_{1}>0.95$ in order to exclude implausible phase functions and to prevent exceedingly lengthy calculations (for large $\left.g_{1}\right)$. For this minimization, Brent's method was used [43].

Then, conversely, $g_{1}$ was fixed at its new value, and $p$ and $h$ were varied to minimize the function

$$
\Delta P(p, h)= \begin{cases}\sum_{i=1}^{567}\left[P_{\mathrm{c}, i}(p, h)-P_{\mathrm{m}, i}\right]^{2}, & p \text { and } h \text { meaningful }, \\ \Delta P_{\text {large }} & \text { otherwise }\end{cases}
$$

where $P=\sqrt{Q^{2}+U^{2}} / I$. "Meaningful" means that the combination of $p$ and $h$ assures that the trial parameterized matrix is a PSM. For this two-dimensional minimization, Powell's method [43] was used, using Brent's method for the one-dimensional subminimizations.

This process was iterated until the values of both $\Delta I$ and $\Delta P$ did not change by more than 0.0001 times their previous values from one iteration to the next.

\subsection{Results and discussion}

The elements of the retrieved PSMs as functions of scattering angle are shown as dashed curves in Figs. 6 and 7. At first sight, the retrieved elements seem quite reasonable, especially $a_{1}(\Theta)$ and $-b_{1}(\Theta) / a_{1}(\Theta)$, although it stands out that in the second case study, the retrieved function $-b_{1}(\Theta) / a_{1}(\Theta)$ reaches a significantly lower maximum than the original one. Also, in both case studies, the retrieved value of $h$ is equal to 1 . Note that in our parameterization, $a_{3}(\Theta) / a_{2}(\Theta)$ and $b_{2}(\Theta)$ are fixed. Since $b_{2}(\Theta) \equiv 0$ in both case studies, there was no need to parameterize $a_{4}(\Theta)$. Hence there are no dashed curves for this element. The original $b_{2}(\Theta) / a_{1}(\Theta)$ and $a_{4}(\Theta) / a_{1}(\Theta)$ are shown for completeness.

The results turned out to be insensitive to the initial guesses for the free parameters. However, sometimes it occurred that the minimizing algorithm let $g_{1}$ wander off to 0.3 or 0.95 . Such solutions 
Table 2

Comparison of original scattering matrices and retrieved PSMs. (See text for further information)

\begin{tabular}{lllllll}
\hline & \multicolumn{2}{l}{ Case study } & $1-$ aggregates & & & \multicolumn{2}{l}{ Case study 2 - spheroids } \\
\cline { 2 - 3 } \cline { 5 - 6 } & Original & Retrieved & & & Original & Retrieved \\
\hline$\langle\cos \Theta\rangle=g_{1}$ & 0.6989 & 0.6910 & & 0.7915 & 0.8181 \\
$-b_{1}\left(90^{\circ}\right) / a_{1}\left(90^{\circ}\right)=p$ & 0.9078 & 0.9095 & & & 0.6856 & 0.6021 \\
Maximum of $-b_{1}(\Theta) / a_{1}(\Theta)$ & 0.9160 & 0.9095 & & & 0.7500 & 0.6021 \\
Location of Maximum & $\Theta=94^{\circ}$ & $\Theta=90^{\circ}$ & & & $\Theta=103^{\circ}$ & $\Theta=90^{\circ}$ \\
$a_{2}\left(90^{\circ}\right) / a_{1}\left(90^{\circ}\right)=h$ & 0.9460 & 1.0000 & & & 0.9129 & 1.0000 \\
$a_{2}\left(180^{\circ}\right) / a_{1}\left(180^{\circ}\right)$ & 0.9306 & 1.0000 & & & 0.9648 & 1.0000 \\
\hline
\end{tabular}

are obviously wrong, and were remedied by choosing different initial guesses for the free parameters.

Quantitatively, the retrieved PSMs are also similar to the original matrices; some interesting comparisons are made in Table 2. Especially the retrieved values of $g_{1}$ differ very little from the original values of $\langle\cos \Theta\rangle$.

As an example, one might now employ Mie theory for interpreting the retrieved parameters. For instance, the retrieved $g_{1}$ in the first case study is reproduced by an ensemble of spheres with refractive index $1.33+0 \mathrm{i}$ that obey a gamma-distribution with an effective size parameter of about 2.25 and an effective variance of 0.09 . However, for this ensemble, $-b_{1}(\Theta) / a_{1}(\Theta)$ never exceeds 0.54. Similar results were obtained for effective variances of 0.03 and 0.20 . High enough values of $-b_{1}(\Theta) / a_{1}(\Theta)$ are obtained, however, if the refractive index is decreased to about $1.20+0 \mathrm{i}$. Since this is an uncommon refractive index for particles suspended in air, we may now infer that the particles in our model atmosphere are not spherical, if in addition we assume that the optical thickness and the albedo for single scattering were correctly assumed. For the second case study, we arrive at a similar result.

The discrepancy between the original and retrieved functions $-b_{1}(\Theta) / a_{1}(\Theta)$ may be caused by the asymmetry of the original functions $-b_{1}(\Theta) / a_{1}(\Theta)$, which have their maxima at scattering angles larger than $90^{\circ}$. Also, it is possible that better results may be obtained with a parameterization of the phase function $a_{1}(\Theta)$ other than a Henyey-Greenstein function. Further studies for other types of parameterizations and other atmospheres are required to find out in detail how one may obtain valuable information about the single-scattering properties of atmospheric particles.

\section{Acknowledgements}

We wish to thank M.I. Mishchenko for comments on an earlier version of this paper. We also thank H. Volten for providing the measurement results shown in Fig. 2. This work has been supported in part by a Columbia University research program funded by NASA Goddard Institute for Space Studies. 


\section{References}

[1] Gehrels T, editor. Planets, stars and nebulae studied with photopolarimetry. Tucson: University of Arizona Press, 1974.

[2] Hansen JE, Hovenier JW. Interpretation of the polarization of Venus. J Atmos Sci 1974;31:1137-60.

[3] Mishchenko MI, Travis LD. Satellite retrieval of aerosol properties over the ocean using measurements of reflected sunlight: effect of instrumental errors and aerosol absorption. J Geophys Res 1997;102:13,543-53.

[4] Mishchenko MI, Travis LD. Satellite retrieval of aerosol properties over the ocean using polarization as well as intensity of reflected sunlight. J Geophys Res 1997;102:16,989-7013.

[5] Van de Hulst HC. Light scattering by small particles. New York: Wiley, 1957, also: New York: Dover, 1981.

[6] Hovenier JW, Van der Mee CVM. Fundamental relationships relevant to the transfer of polarized light in a scattering atmosphere. Astron Astrophys 1983;128:1-16.

[7] Kawabata K, Coffeen DL, Hansen JE, Lane WA, Sato M, Travis LD. Cloud and haze properties from Pioneer Venus polarimetry. J Geophys Res 1980;85:8129-40.

[8] Sato M, Travis LD, Kawabata K. Photopolarimetry analysis of the Venus atmosphere in polar regions. Icarus 1996;124:569-85.

[9] Knibbe WJJ, De Haan JF, Hovenier JW, Travis LD. A biwavelength analysis of Pioneer Venus polarization observations. J Geophys Res 1997;102:10,945-57.

[10] Knibbe WJJ, De Haan JF, Hovenier JW, Travis LD. Analysis of temporal variations of the polarization of Venus observed by Pioneer Venus Orbiter. J Geophys Res 1998;103:8557-74.

[11] Van de Hulst HC. Multiple light scattering: tables, formulas, and applications. New York: Academic Press, 1980.

[12] Irvine WM. Multiple scattering by large particles. Astrophys J 1965;142:1563-75.

[13] Tomasko MG, Smith PH. Photometry and polarimetry of Titan: Pioneer 11 observations and their implications for aerosol properties. Icarus 1982;51:65-95.

[14] West RA, Lane AL, Hart H, Simmons KE, Hord CW, Coffeen DL, Esposito LW, Sato M, Pomphrey RB. Voyager 2 photopolarimeter observations of Titan. J Geophys Res 1983;88:8699-708.

[15] Stammes P. On the interpretation of Titan's intensity and polarization phase curve. In: Kaldeich B, compiler. Proceedings Symposium on Titan, Toulouse, France, 9-12 September 1991, ESA SP-338. Noordwijk, Netherlands: ESA Publications Division, 1992. p. 389-395.

[16] Fry ES, Kattawar GW. Relationships between elements of the Stokes matrix. Appl Opt 1981;20:2811-4.

[17] Hovenier JW, Van de Hulst HC, Van der Mee CVM. Conditions for the elements of the scattering matrix. Astron Astrophys 1986;157:301-10.

[18] Henyey LG, Greenstein JL. Diffuse radiation in the galaxy. Astrophys J 1941;93:70-83.

[19] Hovenier JW. Multiple scattering of polarized light in planetary atmospheres. Astron Astrophys 1971;13:7-29.

[20] Hansen JE. Multiple scattering of polarized light in planetary atmospheres. Part II. Sunlight reflected by terrestrial water clouds. J Atmos Sci 1971;28:1400-26.

[21] Hansen JE, Travis LD. Light scattering in planetary atmospheres. Space Sci Rev 1974;16:527-610.

[22] Tomasko MG, Doose LR. Polarimetry and photometry of Saturn from Pioneer 11: Observations and constraints on the distribution and properties of cloud and aerosol particles. Icarus 1984;58:1-34.

[23] West RA, Sato M, Hart H, Lane AL, Hord CW, Simmons KE, Esposito LW, Coffeen DL, Pomphrey RB. Photometry and polarimetry of Saturn at 2640 and $7500 \AA$ A. J Geophys Res 1983;88:8679-97.

[24] Smith PH, Tomasko MG. Photometry and polarimetry of Jupiter at large phase angles. II. Polarimetry of the South Tropical Zone, South Equatorial Belt, and the polar regions from the Pioneer 10 and 11 missions. Icarus 1984;58:35-73.

[25] Mishchenko MI, Hovenier JW. Depolarization of light backscattered by randomly oriented nonspherical particles. Opt Lett 1995;20:1356-8.

[26] Hovenier JW, Van der Mee CVM. Basic relationships for matrices describing scattering by small particles. In: Mishchenko MI, Hovenier JW, Travis LD, editors. Light scattering by nonspherical particles: theory, measurements, and applications. San Diego: Academic Press, 2000. p. 61-85.

[27] Hovenier JW, Van der Mee CVM. Testing scattering matrices: A compendium of recipes. JQSRT 1996; 55:649-61. 
[28] De Haan JF, Bosma PB, Hovenier JW. The adding method for multiple scattering calculations of polarized light. Astron Astrophys 1987;183:371-91.

[29] Mishchenko MI, Hovenier JW, Travis LD. Concepts, terms, notation. In: Mishchenko MI, Hovenier JW, Travis LD, editors. Light scattering by nonspherical particles: theory, measurements, and applications. San Diego: Academic Press, 2000. p. 3-27.

[30] Szegő G. Orthogonal polynomials, 4th ed. Providence: American Mathematical Society, 1975.

[31] Mishchenko MI, Wiscombe WJ, Hovenier JW, Travis LD. Overview of scattering by nonspherical particles. In: Mishchenko MI, Hovenier JW, Travis LD, editors. Light scattering by nonspherical particles: theory, measurements, and applications. San Diego: Academic Press, 2000. p. 29-60.

[32] Mishchenko MI. Asymmetry parameters of the phase function for densely packed scattering grains. JQSRT 1994;52:95-110.

[33] Mishchenko MI, Macke A. Asymmetry parameters of the phase function for isolated and densely packed spherical particles with multiple internal inclusions in the geometric optics limit. JQSRT 1997;57:767-94.

[34] Volten H, Muñoz O, Rol E, De Haan JF, Vassen W, Hovenier JW, Muinonen K, Nousianen T. Scattering matrices of mineral aerosol particles at $441.6 \mathrm{~nm}$ and $632.8 \mathrm{~nm}$. J Geophys Res, submitted for publication.

[35] Meakin P. The Vold-Sutherland and Eden models of cluster formation. J Colloid Interface Sci 1983;96:415-24.

[36] Bar-Nun A, Kleinfeld I, Ganor E. Shape and optical properties of aerosols formed by photolysis of acetylene, ethylene, and hydrogen cyanide. J Geophys Res 1988;93:8383-7.

[37] Scattergood TW, Lau EY, Stone BM. Titan's aerosols. I. Laboratory investigations of shapes, size distributions, and aggregation of particles produced by UV photolysis of model Titan atmospheres. Icarus 1992;99:98-105.

[38] Draine BT, Flatau PJ. Discrete-dipole approximation for scattering calculations. J Opt Soc Am A 1994;11:1491-9.

[39] Draine BT. The discrete dipole approximation for light scattering by irregular targets. In: Mishchenko MI, Hovenier JW, Travis LD, editors. Light scattering by nonspherical particles: theory, measurements, and applications. San Diego: Academic Press, 2000. p. 131-45.

[40] Mishchenko MI, Travis LD. Capabilities and limitations of a current FORTRAN implementation of the $T$-matrix method for randomly oriented, rotationally symmetric scatterers. JQSRT 1998;60:309-24.

[41] Mishchenko MI, Travis LD, Macke A. T-matrix method and its applications. In: Mishchenko MI, Hovenier JW, Travis LD, editors. Light scattering by nonspherical particles: theory, measurements, and applications. San Diego: Academic Press, 2000. p. 147-72.

[42] Hansen JE. Multiple scattering of polarized light in planetary atmospheres. Part I. The doubling method. J Atmos Sci 1971;28:120-5.

[43] Press WH, Teukolsky SA, Vetterling WT, Flannery BP. Numerical recipes: the art of scientific computing, 2nd ed.. Cambridge: Cambridge University Press, 1992. 